\title{
Lectoescritura Inicial y su Discurso Pedagógico en el Currículo Nicaragüense
}

\author{
MEZA-DURIEZ, Rafael Santiago ${ }^{1}$
}

\section{RESUMEN}

Este artículo resume elementos de una investigación que se propuso inferir el discurso pedagógico que subyace al método Fonético, Analítico y Sintético (FAS), tal como se aplica en Nicaragua como elemento fundamental del currículo y la política educativa para la enseñanza de la lectura y escritura. Se determinaron los elementos conceptuales que constituyen al discurso pedagógico como constructo teórico. Estos elementos fueron identificados en el currículo vigente para reconstruir el discurso pedagógico subyacente y determinar si este último es de tipo reproductor o transformador. El estudio se realizó mediante el análisis de un cuerpo de documentos curriculares de diferentes rangos jerárquicos. Su principal contribución es que revela el carácter reproductor del FAS como método insignia de la política nacional y, por tanto, su incapacidad para cerrar las brechas históricas de equidad que han sido sostenidas en el tiempo por el sistema educativo nicaragüense.

Educación básica. Sociología de la educación. Alfabetización.

\section{Early grades literacy and its pedagogical discourse on the nigaraguan curriculum}

\section{ABSTRACT}

This article resumes the findings of a research whose aim was to identify the pedagogical discourse underlying Phonics, Analytics and Synthetic method (FAS), which is the method implemented on the Nicaraguan curriculum as an essential element of the public policy on early grades literacy instruction. The conceptual elements that constitute the pedagogical discourse as a theoretical construct were explained. Those same elements were later identified on the national curriculum in order to make visible the underlying pedagogical discourse and to determine whether or not, its nature was reproductive or transformative. A documentary analysis was carried out on a corpus of curricular documents within a specific hierarchy. As its main contribution, this research reveals the reproductive nature of FAS and its inability to close in the historical equity gaps upheld for a long time by the Nicaraguan education system.

\footnotetext{
${ }^{1}$ Professor no Departamento de Educação e Mestrando em Educação na Universidade CentroAmericana - Manágua, Nicarágua. E-mail: santiagoduriezm@gmail.com. Orcid: http://orcid.org/0000-0003-1920-5180.
} 
Basic education. Sociology of education. Literacy.

\section{Alfabetização inicial e seu discurso pedagógico no currículo nicaraguense}

\section{RESUMO}

Este artigo sintetiza elementos de uma pesquisa que se propôs a inferir o discurso pedagógico que fundamenta o método Fonético, Analítico e Sintético (FAS), aplicado na Nicarágua como elemento fundamental do currículo e da política educacional para o ensino da leitura. e escrever. Foram determinados os elementos conceituais que constituem o discurso pedagógico como construção teórica. Esses elementos foram identificados no currículo atual para reconstruir o discurso pedagógico subjacente e determinar se este é reprodutivo ou transformador. $O$ estudo foi realizado por meio da análise de um corpo de documentos curriculares de diferentes níveis hierárquicos. Sua principal contribuição é que revela a natureza reprodutiva da FAS como o principal método da política nacional e, portanto, sua incapacidade de fechar as lacunas históricas de eqüidade que foram sustentadas ao longo do tempo pelo sistema educacional nicaraguense.

Educação Básica. Sociologia da educação. Alfabetização.

\section{INTRODUCCIÓN}

Aprender a leer y escribir es la piedra angular sobre la que se erige cualquier sistema educativo. Hay un prolífico cuerpo de investigación respecto a la lectoescritura inicial (LEI). En términos generales, se han logrado avances significativos en este campo durante la última década (KIM, BOYLE, ZUILKOWSKI; NAKAMURA, 2016). Sin embargo, existen todavía evidentes brechas de investigación alrededor de lo que en realidad funciona para enseñar a leer y a escribir en contextos como el de Nicaragua, en donde la pobreza y la falta de equidad afecta de manera significativa las posibilidades de niños y niñas para acceder a una educación de calidad (Programa de Capacidades Lac READS [PCRL], 2016).

Banerjee y Dufflo (2012) explican que el problema de las políticas educativas a nivel mundial es que no responden a las necesidades del contexto, y suelen ser diseñadas "pensando en la élite más que en los niños que asisten habitualmente al colegio" (p.127). Efectivamente, la pobreza extrema, la falta de equidad, la diversidad cultural y las desigualdades sociales son condiciones habituales que un sistema educativo debe enfrentar en países en vías de desarrollo. Sin embargo, la evidencia basada en ensayos aleatorios controlados 
(BANERJEE; DUFFLO, íbid) permite determinar lo que funciona en las aulas de clases, y valorar la alternativa que mejor se corresponda con las características locales. En otras palabras, el contexto puede ayudarnos a construir un sistema educativo "a la medida". Esta visión es fundamental para crear agencia educativa.

Sin embargo, muchas de las intervenciones en LEl a lo largo del mundo se suelen ejecutar desde la visión del "western paradigm" (WAGNER, MURHPY; KORNE, 2012, MOUNT-COURS, 2016). En ese sentido la enseñanza de la LEI a lo largo de la región latinoamericana está provista de los sesgos propios de una visión onto-epistemológica que, si bien arroja varias luces para entender cómo la niñez aprende a leer y a escribir, no termina de comprender las influencias de la diversidad cultural en los aprendizajes de niños y niñas (SALAZAR PÉREZ; SAAVEDRA, 2017). De ahí entonces la importancia de determinar los discursos pedagógicos (BERNSTEIN, 1975a, 1975b, 1975c, 1990) que subyacen a las políticas de enseñanza de la LEl y que son expresadas en los currículos.

En Nicaragua, país que ha sido beneficiado por diversas propuestas de intervención educativa, se hace necesario determinar el discurso pedagógico subyacente en la enseñanza de la lectoescritura inicial. En nuestro país no existe una política pública educativa sino más bien políticas de gobierno e incluso, de administraciones (CASTILLO, ELVIR; VIJIL, 2016). Todo esto trae como consecuencia que la calidad de los aprendizajes en LEI sea insuficiente, lo que se refleja en las pruebas TERCE (IEEPP, 2016). Estas invitan a pensar que la niñez nicaragüense sigue aprendiendo desde una óptica de reproducción de contenidos, sin criticidad, sin poder expresar quien son en realidad y sin habilidades de nivel superior, como el pensamiento crítico, capacidad de abstracción y comprensión lectora entre otras.

Para comprender esta situación, es necesario analizar con detenimiento la política actual de LEI y como configura el discurso pedagógico (BERNSTEIN, 1990) que regula los procesos de enseñanza. En ese sentido, el método Fonético, Analítico y Sintético (FAS,) en tanto lineamiento estratégico por excelencia del Ministerio de Educación para enseñar LEl, adquiere una relevancia fundamental, ya que toda la política nacional en esta materia se basa en la implementación del método. Por ende, los paradigmas subyacentes al mismo deben ser analizados a la luz de la teoría sociocultural, y de los principios de equidad e inclusión educativa para así determinar si su naturaleza es de tipo reproductora o transformadora. En consecuencia, esta investigación se propuso responder a la siguiente pregunta: ¿Cuál es el discurso pedagógico que subyace a las políticas educativas para la enseñanza de la lectoescritura inicial ejecutadas por la implementación del Método FAS? 


\section{Un marco referencial para la determinación del discurso pedagógico}

La teoría sociocultural de la educación: el discurso reproductor y el transformador

Para comprender el discurso pedagógico que subyace a la enseñanza de la LEl, es fundamental explicar esta acepción partiendo del concepto básico que nos brinda Bernstein (BERNSTEIN; DÍAZ, 1985):

Es posible definir el discurso pedagógico como las reglas que regulan la producción, reproducción y cambio de lo que cuenta como textos pedagógicos legítimos. En ese sentido, el discurso pedagógico no sería un repertorio de contenidos (conceptos, teorías o conjunto de enunciados, conjunto de presentaciones), sino una gramática de producción de textos para su distribución y reproducción en el contexto de recontextualización.

El discurso pedagógico determina las reglas y principios con que se debe ejercer el poder en los procesos de enseñanza - aprendizaje y los tres elementos que conforman el sistema de mensaje son currículo, pedagogía y evaluación. Apple (2017) reconoce que pueden existir dos tipos de discursos, uno reproductor de las hegemonías que conforman la sociedad, y otro, de tipo transformador, en el cual no solo se resiste a la imposición de una cultura específica, sino que se crean las condiciones para su transformación.

Desde esta perspectiva es importante trasladarnos al campo de la sociología de la educación. Diversos autores (BOURDIEU; PASSERON, 2000, BERNSTEIN 1975A, 1975B, 1975C, 1990, APPLE, 2017, 2019; PERRENOUD, 2008) han estudiado las relaciones entre procesos educativos, cultura, sociedad y ejercicio de poder. No obstante, es el estructuralismo de Bourdieu el que empezó a proponer una relación dialéctica entre estos elementos (ASTETE BARRENECHEA, 2017). Para Bourdieu las escuelas ejercen una violencia simbólica. En palabras del mismo autor, "toda acción pedagógica es, objetivamente, violencia simbólica por cuanto es la imposición de un arbitrario cultural por parte de un poder arbitrario" (BOURDIEU; PASSERON, trad. 2000, p.5). Como se puede apreciar, Bourdieu nos brinda los elementos para comprender el discurso pedagógico en su dimensión reproductora de las condiciones hegemónicas y formas producción cultural, social y económica.

Por otra parte, autores como Freire (2012) y Hooks (1994, 2003, 2010) identifican en la acción pedagógica, una práctica de liberación y transformación de la persona, así como de las condiciones materiales en que esta se encuentra. Freire reconoce la existencia de una concepción bancaria de la educación que funge como herramienta de opresión y ubica a docentes y estudiantes en posiciones antagónicas de opresores y oprimidos. En su discurso, Freire 
determina la importancia de reconocer los roles de docentes y estudiantes; señala que ambos ejercen roles claros (el o la docente se encarga de crear las condiciones y experiencias de aprendizaje para leer el mundo y contrastar teoría y práctica; mientras que el o la estudiante se encargan de enriquecer, desde su visión, un diálogo de saberes en el cual expresan "su propia palabra") pero en una relación horizontal.

El discurso transformador de Freire ha sido reinterpretado por Hooks (2010), quien sostiene que la pedagogía de la libertad implica una labor constante para enseñar a pensar críticamente a través de una acción pedagógica que motive al estudiantado a desarrollar esta competencia; pero que también oriente a pensar en nuevas formas de dominación que no estaban contempladas en el discurso inicial de Freire, como aquella que ejerce el hombre sobre la mujer (HOOKS, 1994) También señala la importancia de incorporar en la educación las visiones de grupos marginados y excluidos, y emplear recursos como la incorporación de las narrativas propias como punto de partida para comprender la realidad (HOOKS, 2003).

El criterio fundamental para determinar si el discurso pedagógico es de carácter reproductor o transformador, es el de equidad e inclusión educativa. Apple (2017) destaca que el análisis crítico de las hegemonías incorporadas al currículo, requiere, más que detectar los elementos presentes en la narrativa, identificar "las ausencias presentes" (p. 4) en ella y que invisibilizan la diversidad cultural, lo que coloca en situación de desigualdad a las personas que se salen del ideario hegemónico. Por su parte, Delgado Montoya (2007) reconoce que los sistemas educativos carecen de inclusión y equidad en la medida que se segrega esa diversidad cultural. Este autor plantea que, a pesar que hay una integración simbólica producto de un mayor acceso a los bienes simbólicos del mundo globalizado, los sistemas educativos tienden a obviar la integración social y material. La diversidad étnica y cultural no son tomadas en consideración, así como tampoco se incluyen las condiciones socioeconómicas en que se desarrolla la persona. Un sistema educativo con inclusión y equidad es aquél que es capaz de ver la diversidad en términos de oportunidades de aprendizaje y no de déficits.

Los elementos constitutivos del discurso pedagógico

Si bien todo lo anterior nos permite comprender los criterios para valorar si un discurso es de tipo reproductor o transformador, la abstracción con la que se han construido tales categorías requiere aún de la constitución de un marco de análisis más concreto. En ese sentido, hemos realizado una relectura de los elementos constitutivos del discurso pedagógico definidos por Bernstein (1975a, 1975b, 1975c, 1990) que servirá como punto de encuentro y síntesis de las 
teorías estudiadas. Para Bernstein el discurso pedagógico tiene cuatro elementos centrales, el lenguaje, las reglas regulativas de jerarquía o discurso regulativo y las reglas instruccionales o discurso instruccional, y las formas de evaluación. Cada uno de estos elementos se expresan de diferentes maneras según se trate de un discurso reproductor o transformador. Esto se detalla en la tabla 1.

Tabla 1: Elementos constituyentes del discurso pedagógico

\begin{tabular}{|c|c|c|}
\hline $\begin{array}{l}\text { Elementos del } \\
\text { discurso }\end{array}$ & $\begin{array}{l}\text { Discurso pedagógico de tipo } \\
\text { reproductor }\end{array}$ & $\begin{array}{l}\text { Discurso pedagógico de tipo } \\
\text { transformador }\end{array}$ \\
\hline Lenguaje & $\begin{array}{l}\text { Elaborado: } \\
\text { 1.-Uso de variantes sintácticas } \\
\text { complejas. } \\
\text { 2.-Uso del lenguaje escolar en } \\
\text { detrimento del lenguaje propio del } \\
\text { entorno de procedencia. } \\
\text { 3.-Poco significado en tanto no } \\
\text { hay recuperación de } \\
\text { conocimientos previos }\end{array}$ & $\begin{array}{l}\text { Elaborado y restringido } \\
\text { 1.-Variantes sintácticas } \\
\text { reconocibles como punto de partida } \\
\text { enseñar más complejas. } \\
\text { 2.-El lenguaje de procedencia es el } \\
\text { punto de partido para enseñar el } \\
\text { lenguaje escolar } \\
\text { 3.-Incorporación de conocimientos } \\
\text { previos }\end{array}$ \\
\hline \multirow[t]{2}{*}{$\begin{array}{c}\text { Reglas } \\
\text { Regulativas } \\
\text { de jerarquía: }\end{array}$} & $\begin{array}{l}\text { Enmarcación Fuerte: } \\
\text { 1.-Explícitamente se regulan los } \\
\text { procesos de interacción Docente } \\
\text { estudiante. } \\
\text { 2.-El proceso de interacción es } \\
\text { regulado exclusivamente por el } \\
\text { docente. } \\
\text { 3.-Se define lo que se puede o no } \\
\text { se puede decir (Performatividad, } \\
\text { subjetivación e inteligibilidad } \\
\text { restrictivas }\end{array}$ & $\begin{array}{l}\text { Enmarcación Débil: } \\
\text { 1.-Hay una regulación implícita de } \\
\text { los procesos de interacción. } \\
\text { 2.-El control del proceso de } \\
\text { interacción recae en los } \\
\text { estudiantes y su ritmo de } \\
\text { aprendizaje. } \\
\text { 3.-Es difusa la línea de lo que se } \\
\text { debe aprender y marcada por } \\
\text { intereses y necesidades del } \\
\text { estudiantado. } \\
\text { 4.-El aprendizaje se crea entre } \\
\text { todos y en libertad a partir del } \\
\text { diálogo y desde sus respectivos } \\
\text { roles. }\end{array}$ \\
\hline & $\begin{array}{l}\text { Clasificación Fuerte: } \\
\text { 1.-Currículo agregado } \\
\text { 2.-Contenidos con relación } \\
\text { cerrada y aislados entre sí. } \\
\text { 3.-Jerarquías marcadas entre } \\
\text { asignaturas, agentes y agencias } \\
\text { de control simbólico. }\end{array}$ & $\begin{array}{l}\text { Clasificación débil: } \\
\text { 1.-Currículo integrado. } \\
\text { 2.-Contenidos con relación abierta } \\
\text { entre sí. } \\
\text { 3.-Jerarquías difusas entre } \\
\text { asignaturas, agentes y agencias. } \\
\text { 4.-Integración de las tareas. }\end{array}$ \\
\hline
\end{tabular}




\begin{tabular}{c|l|l}
\hline & $\begin{array}{l}\text { 4.-Fuerte especialización de las } \\
\text { tareas. }\end{array}$ & $\begin{array}{l}\text { 5.-Incluye diversidad, es posible } \\
\text { adaptar contenidos a necesidades } \\
\text { específicas de aprendizaje. }\end{array}$ \\
\hline $\begin{array}{c}\text { Reglas } \\
\text { instruccional } \\
\text { es: } \\
\text { secuencia, } \\
\text { ritmo, } \\
\text { selección, } \\
\text { criterios. }\end{array}$ & $\begin{array}{l}\text { Se seleccionan y se imponen los } \\
\text { temas, los ritmos y tiempos de } \\
\text { aprendizaje, así como los criterios } \\
\text { de evaluación. No hay posibilidad } \\
\text { de error. }\end{array}$ & $\begin{array}{l}\text { Se negocian los contenidos a partir } \\
\text { de ciertos estándares, los procesos } \\
\text { no son graduados, se respetan los } \\
\text { ritmos, se reflexionan sobre los } \\
\text { criterios de evaluación y se hace } \\
\text { del error una oportunidad de } \\
\text { aprendizaje. }\end{array}$ \\
\hline $\begin{array}{c}\text { Evaluación } \\
\text { objetivos del currículo. Énfasis en }\end{array}$ & $\begin{array}{l}\text { lo medible y sumativo. Crea } \\
\text { representaciones del buen } \\
\text { alumno y del mal alumno. }\end{array}$ & $\begin{array}{l}\text { Enfocada en los aprendizajes. } \\
\text { estudiante. Se fundamenta en el } \\
\text { concepto de zona de desarrollo } \\
\text { próximo. }\end{array}$ \\
\hline
\end{tabular}

Fuente: Elaboración propia a partir de Bernstein (1975a, 1975b, 1975c, 1990).

\section{La lectoescritura inicial desde el enfoque cultural}

El discurso que ubica a la LEI como práctica sociocultural, posee unos principios a los cuales deberá regirse el discurso pedagógico y sus elementos. Estos principios han sido definidos por Frankel, Rowe, Becker; Pearson (2015) quienes plantean criterios para entender la LEI desde este enfoque:

- Leer y escribir es un proceso constructivo, integrador y crítico que se sitúa en unas prácticas sociales concretas: Es decir que se compone de relaciones complejas entre persona que lee, texto, contexto y actividades de aprendizajes.

- La lectura fluida es moldeada por los procesos del lenguaje y los contextos: La fluidez se desarrolla paralelamente con otras habilidades del lenguaje como la escritura, el habla y la escucha, e incorpora otros indicadores de dominio como la prosodia, la automaticidad y la precisión al leer.

- Saber leer y escribir es estratégico y disciplinario: Además de enseñar las estrategias de lectura y escritura para su desarrollo, éstas deben enseñarse en el marco de disciplinas que ayuden a situar las prácticas sociales en un contexto, y no como habilidades aisladas del contexto del que provienen.

- Saber leer y escribir implica motivación, compromiso y voluntad: El aprendizaje de la LEI debe partir desde la motivación intrínseca del estudiantado. 
- Saber leer y escribir es una habilidad que se continúa desarrollando para toda la vida: El aprendizaje de la LEI debe ofrecer las herramientas para su profundización a lo largo de la vida de la persona que lee, de acuerdo con sus motivaciones y propósitos.

Adicional a ello, hemos incorporado como elemento de análisis al marco conceptual denominado Diseño Universal de Aprendizaje (DUA) (PILGRIM; KRIS WARD, 2017). EI DUA es marco de referencia para la instrucción inclusiva. El modelo se fundamenta en la teoría de Vigotsky ya que tiene como piedra angular la zona de desarrollo próximo. Precisamente, el DUA propone el desarrollo de andamiajes adaptativos a las necesidades y que procuren llevar al niño o la niña al máximo nivel posible de aprendizaje. En ese sentido, este modelo cuenta con tres principios o redes de trabajo, cada una con sus propias líneas directrices, mismas que se detallan a continuación:

Tabla 2: Principios del Diseño Universal de Aprendizaje y sus líneas directrices

\begin{tabular}{l|l}
\hline Principios & Líneas directrices \\
\hline Reconocimiento & $\begin{array}{l}\text { Opciones de percepción: presentar el conocimiento y el aprendizaje en } \\
\text { formatos que se ajusten a las necesidades del estudiantado. }\end{array}$ \\
\cline { 2 - 3 } & $\begin{array}{l}\text { Planificar formas inclusivas de introducir nuevo vocabulario y símbolos, } \\
\text { a través de distintos formatos. }\end{array}$ \\
\cline { 2 - 3 } & $\begin{array}{l}\text { Enfocarse en la comprensión profunda del tema: activar conocimientos } \\
\text { previos, asistir a estudiantes con el procesamiento de la información, } \\
\text { fortalecer la memoria y la transferencia del conocimiento a resolución } \\
\text { de problemas. }\end{array}$ \\
\hline \multirow{2}{*}{ Estratégico } & $\begin{array}{l}\text { Resolver problemas haciendo uso del espacio pedagógico, } \\
\text { trascendiendo del típico formato de papel y lápiz }\end{array}$ \\
\cline { 2 - 3 } & $\begin{array}{l}\text { Abastecer al estudiantado de diversos formatos para promover la } \\
\text { comunicación. Proveer herramientas y andamios para enseñar a } \\
\text { comunicar efectivamente hasta que haya una autorregulación }\end{array}$ \\
\cline { 2 - 3 } & $\begin{array}{l}\text { Reforzar las funciones ejecutivas. Modelar la organización, la } \\
\text { concentración, el uso de la memoria, la resolución de problemas. }\end{array}$ \\
\hline Afectivo & $\begin{array}{l}\text { Promover la elección individual de las rutas de aprendizaje, y apartar y } \\
\text { distractores del aprendizaje. }\end{array}$ \\
\cline { 2 - 3 } & $\begin{array}{l}\text { Fomentar y alentar la persistencia. Recordar las metas de aprendizaje, } \\
\text { monitorear la frustración y ajustar el nivel si es necesario. }\end{array}$ \\
\cline { 2 - 3 } & $\begin{array}{l}\text { Promover la autorregulación en el estudiantado. } \\
\text { Fuboración propia a partir de Pilgrim; Kris Ward (2017). }\end{array}$ \\
\hline
\end{tabular}


Así pues, con base en el enfoque sociocultural de la lectura y modelo DUA, es posible explicar el marco que denominamos "las $5 \mathrm{~T}$ " y que se basa en los trabajos de Allington (2002) y Kim, Boyle, et. al (2016).

- Teaching o enfoque enseñanza: Allington reconoce que los profesores efectivos y ejemplares son aquellos que enseñan explícitamente habilidades específicas de lectoescritura inicial. Destaca igualmente la importancia de transferir poco a poco responsabilidades a sus estudiantes en aras de buscar su independencia y que se conviertan en lectores auto regulados. Ello se relaciona con el modelo instruccional de Pearson y Gallagher (BRAOJOS; PÉREZ, 2012) en donde se combinan fases de modelaje o demostración directa, con práctica guiada y práctica autónoma. Las estrategias de enseñanza deben fomentar tanto las habilidades restringidas (aquellas que están referidas a la decodificación, deletreo y fluidez), como las amplias (vocabulario y comprensión) (PARIS, 2005) desde un enfoque balanceado (GUARDIA, 2018). Deben realizarse sobre la base del diálogo y la interacción horizontal (FREIRE, 2002;) en entornos motivantes y afectivos que ayuden a construir significado (HOOKS, 1993) y que fomenten la zona de desarrollo próximo. Se deben recuperar prácticas letradas y culturales del entorno cercano, como la familia o la comunidad, y vincularlas con la vida en las escuelas (JACOBS, 2017). Es importante también partir del nivel sociolingüístico restringido de niños y niñas para apoyarles en la construcción de estructuras lingüísticas más elaboradas, lo que a larga deriva en un pensamiento más universal, complejo e independiente del contexto.

- Tasks o Actividades de aprendizaje: Allington reconoce que las actividades de aprendizaje, lejos de ser requisitos o mero trámites expeditos para completar el día de clases, deben ser prolongadas en el tiempo y facilitar a los estudiantes diversas opciones de aprendizaje, así como trabajo interdisciplinario y motivación por aprender. Las actividades de aprendizajes varían según la habilidad que se deba desarrollar. Cuando nos referimos a habilidades, evocamos las 5 básicas definidas por el National Reading Panel a inicio de este siglo: Conciencia fonológica, Principio alfabético, Fluidez, Vocabulario y Comprensión lectora. Según Pilgrim y Kris Ward (2017), la instrucción inclusiva de cada una de estas habilidades debe incorporar los tres principios del DUA y sus correspondientes líneas directrices en aras de garantizar el aprendizaje efectivo y significativo de todo el estudiantado.

- Texts o materiales: Allington esgrime que la niñez, para aprender a leer, no solo necesita grandes cantidades de libros variados y diversos, sino que sean adecuados a su nivel, lo que implica permitir opciones de lectura según las necesidades y motivaciones del niño o de la niña, para que 
puedan aplicar en estos libros las estrategias y habilidades que le muestran sus docentes. No se puede limitar la lectura a libros de textos instructivos 0 manuales de asignatura. En los casos con resultados deficientes en lectoescritura inicial, Allington (2002) encontró que el estudiantado pasaba cuatro horas diarias frente a textos instruccionales que no podían leer apropiadamente por no estar acordes a su nivel. Adicionalmente, Jacobs (2017) indica que las narrativas de las familias, los textos de diversos tipos que estén en el hogar, y las formas cotidianas de acercamiento a la escritura, son recursos válidos de los cuales hacer uso para la enseñanza de la LEI.

- Time o tiempo de instrucción: El tiempo según Allington es un factor determinante para la enseñanza de la lectoescritura inicial. En las aulas efectivas, el estudiantado dedicaba en la mayoría de los casos un $50 \%$ de tiempo efectivo de clases a la lectura y escritura, frente a espacios menos exitosos en donde otros aspectos (generalmente administrativos) ocupaban poco más del $90 \%$ de ese mismo tiempo. También es importante equilibrar entre el ritmo de aprendizaje del o la estudiante, y los períodos críticos para el desarrollo de las habilidades, sobre todo aquellas que son restringidas y referidas al dominio del código escrito, para las cuales hay un momento específico en el primer año de primaria. Es importante que se optimice el tiempo y se evite a toda costa el efecto Matthews (PILGRIM; KRISS WARD, 2017), es decir el ensanchamiento de las brechas entre estudiantes que saben decodificar y deletrear correctamente (por ende, seguirán con un buen ritmo su aprendizaje), y aquellos con retrasos en estas habilidades y que difícilmente podrán alcanzar al resto en cuanto a profundidad del aprendizaje.

- Tests o evaluación del aprendizaje: La evaluación debe ser formativa, y debe ver en el error, la oportunidad para aprender más y mejor. La evaluación debe permitir que el aprendizaje se evidencie en distintos formatos ajustados a las necesidades y particularidades del estudiantado. Se debe trazar una línea de progresión ajustada a la zona real o inicial del o la estudiante y que permita fijar exactamente hasta dónde puede llegar con los andamiajes adecuados.

\section{Metodología}

El enfoque seleccionado para este estudio es el cualitativo con un carácter exploratorio y comparativo. Considerando que el propósito de este estudio es inferir el discurso pedagógico subyacente a la implementación del método FAS, es necesario remitirse a los documentos oficiales que concretaron y orientaron 
la realización de las prácticas pedagógicas durante su implementación. Cada documento oficial fue considerado como una unidad de análisis. En consecuencia, el método seleccionado fue el análisis documental.

Según Ary, Jacobs, Sorensen y Razavieh (2010) este método investigativo permite, entre otras posibilidades, comprender los sesgos e intencionalidades presentes en documentos específicos. Asimismo, destacan que la presencia del observador o investigador no ejerce influencia en el objeto de estudio. Ello debido a que los documentos escritos guardan el pensamiento y los enfoques con que fueron diseñados.

El estudio es de tipo exploratorio ya que el tema no ha sido suficientemente investigado por lo que no se cuenta con fuentes secundarias 0 estudios anteriores. Por otro lado, se trató de incorporar nuevas perspectivas de análisis para valorar y comprender las políticas públicas sobre LEI. El análisis documental permitió extraer, analizar y contrastar los elementos del discurso pedagógico subyacentes a la política seleccionada. Para ello, se elaboró una guía de análisis documental multinivel con el propósito de identificar a lo largo de los documentos a estudiar, determinados indicadores extraídos del marco conceptual. Lo anterior facilitó la reconstrucción del discurso pedagógico subyacente a FAS.

Los niveles que analizó esta guía son los siguientes:

- Nivel estratégico-pedagógico: En este nivel se analizaron documentos generales reguladores que expresan los enfoques pedagógicos, contenidos a estudiar y perfiles terminales (currículos, mallas de contenidos, entre otros).

- Nivel metodológico: En este nivel, se analizaron documentos que presentan estrategias metodológicas para la enseñanza de la LEI (guías para docentes, documentos normativos operativos, etc.). Generalmente en estos documentos se encuentran pautas metodológicas, guías de enseñanza, entre otros.

- Nivel didáctico: En este nivel, se analizaron los recursos didácticos oficiales y definidos para la enseñanza de la LEI. Nos referimos a los libros de texto y demás materiales de aprendizaje.

Se han construido categorías de análisis e indicadores con los cuales analizar los documentos enunciados arriba. La construcción de estas categorías surge a partir de la adaptación de las teorías de Bernstein respecto al Discurso Pedagógico. Esta adaptación es producto de un análisis integrado de sus postulados con el marco referencial de las $5 \mathrm{~T}$, y los principios definidos en el marco conceptual para el diseño universal de aprendizaje.

La triangulación de la información se desarrolló de la siguiente manera: 
- Se definieron los documentos oficiales correspondientes a cada nivel (Estratégico-Pedagógico, Metodológico, Didáctico).

- Se analizó cada documento conforme a las categorías de análisis y los indicadores que corresponden a aquellas.

- Se realizó un análisis de tipo categorial a partir de los elementos constitutivos del Discurso Pedagógico (Bernstein, 1990) para determinar si el carácter predominante en la política es de tipo reproductor 0 transformador.

Una vez realizado este análisis, se procedió a una reconstrucción del discurso pedagógico para la enseñanza de la LEl. Para ello, fue necesario explicitar las intencionalidades del currículo que subyacen a un nivel más profundo de lo que está declarado formalmente. En ese sentido, se organizó el análisis usando como base el marco de las $5 \mathrm{~T}$ para entender los siguientes componentes:

- El enfoque real de la enseñanza de la LEI más allá de lo formalmente declarado y la consistencia de éste con sus orientaciones metodológicas.

- La idoneidad de las actividades de aprendizajes para generar desafíos cognitivos ajustados al nivel real del estudiantado y que recuperen las prácticas de cultura escrita propios del entorno de procedencia.

- La ruta de progresión definidas para el desarrollo de la LEI y las formas en que se evalúa sistemáticamente.

- La significatividad y adecuación al nivel real del estudiantado de los materiales definidos en el currículo.

- Los tiempos efectivos dedicados a la lectura y la escritura y cómo se aprovecha para acercar al estudiantado a estas competencias.

Para el análisis del método FAS se han identificado los elementos constituyentes del discurso pedagógico, a saber: 1) Lenguaje, 2) Discurso regulativo, y 3) Discurso instruccional. Cada uno de esos componentes ha sido analizado en los tres niveles que constituyen la política objeto de estudio. Los documentos analizados son los siguientes:

- Nivel estratégico-pedagógico

- Currículo nacional básico (MINED, 2009).

1. Nivel metodológico.

- Malla curricular de lengua y literatura para ciclo básico (MINED, 2019a, 2019b).

- Orientaciones metodológicas para el método FAS (MINED, 2015a).

2. Nivel didáctico 
- Cuaderno de aprestamiento (MINED, 2015b).

- Libro de texto (MINED, 2019c).

A continuación, presentamos las categorías de análisis:

Tabla 3: Categorías de análisis

\begin{tabular}{|c|c|c|c|}
\hline $\begin{array}{l}\text { Elementos } \\
\text { del Discurso } \\
\text { Pedagógico }\end{array}$ & $\begin{array}{l}\text { Dimensiones del } \\
\text { Marco de las } 5 T \\
\text { involucradas }\end{array}$ & $\begin{array}{l}\text { Categorías de } \\
\text { Análisis }\end{array}$ & Indicadores \\
\hline \multirow{8}{*}{ Lenguaje } & \multirow{8}{*}{$\begin{array}{l}\text { Se incorpora } \\
\text { transversalmente } \\
\text { a todas las } \mathrm{T}\end{array}$} & \multirow{3}{*}{$\begin{array}{l}\text { Formatos de } \\
\text { comunicación }\end{array}$} & $\begin{array}{l}\text { Diversidad de formatos para } \\
\text { presentar la información }\end{array}$ \\
\hline & & & $\begin{array}{l}\text { Formas para expresar o } \\
\text { comunicar los aprendizajes }\end{array}$ \\
\hline & & & $\begin{array}{l}\text { Formas comunicacionales } \\
\text { para orientar y brindar } \\
\text { andamiajes }\end{array}$ \\
\hline & & \multirow{3}{*}{$\begin{array}{l}\text { Reconocimiento } \\
\text { del lenguaje de } \\
\text { procedencia }\end{array}$} & $\begin{array}{l}\text { Transición progresiva de un } \\
\text { lenguaje restringido a uno } \\
\text { elaborado. }\end{array}$ \\
\hline & & & $\begin{array}{l}\text { Definición del tipo de lenguaje } \\
\text { a adquirir }\end{array}$ \\
\hline & & & Variantes sintácticas \\
\hline & & \multirow{2}{*}{$\begin{array}{l}\text { Creación de } \\
\text { significado }\end{array}$} & $\begin{array}{l}\text { Optimización de la elección } \\
\text { individual y autonomía }\end{array}$ \\
\hline & & & $\begin{array}{l}\text { Disminución de inseguridad y } \\
\text { distracciones. }\end{array}$ \\
\hline \multirow{6}{*}{$\begin{array}{l}\text { Discurso } \\
\text { regulativo }\end{array}$} & \multirow{6}{*}{ Teaching } & \multirow{4}{*}{ Clasificación } & $\begin{array}{l}\text { Enfoque definido para la } \\
\text { enseñanza de la LEI }\end{array}$ \\
\hline & & & $\begin{array}{l}\text { Determinación de } \\
\text { conocimientos, habilidades y } \\
\text { valores a aprender }\end{array}$ \\
\hline & & & $\begin{array}{l}\text { Relación de contenidos entre } \\
\text { sí, y con otras materias. }\end{array}$ \\
\hline & & & $\begin{array}{l}\text { Jerarquías y rutas de } \\
\text { progresión definidas en el } \\
\text { currículo }\end{array}$ \\
\hline & & \multirow[t]{2}{*}{ Enmarcación } & $\begin{array}{l}\text { Definición de roles y } \\
\text { posiciones de poder en el } \\
\text { proceso E-A }\end{array}$ \\
\hline & & & $\begin{array}{l}\text { Diálogo, participación activa y } \\
\text { horizontalidad }\end{array}$ \\
\hline
\end{tabular}




\begin{tabular}{|c|c|c|c|}
\hline \multirow{6}{*}{$\begin{array}{l}\text { Discurso } \\
\text { instruccional }\end{array}$} & \multirow{2}{*}{ Tasks, texts } & \multirow{2}{*}{$\begin{array}{l}\text { Reglas de } \\
\text { selección }\end{array}$} & $\begin{array}{l}\text { Diversidad de actividades de } \\
\text { aprendizaje según } \\
\text { necesidades individuales }\end{array}$ \\
\hline & & & $\begin{array}{l}\text { Diversidad de materiales y } \\
\text { recursos según necesidades } \\
\text { individuales }\end{array}$ \\
\hline & \multirow[b]{2}{*}{ Time } & \multirow[b]{2}{*}{$\begin{array}{l}\text { Reglas de } \\
\text { secuencia y ritmo }\end{array}$} & $\begin{array}{l}\text { Flexibilidad en los ritmos de } \\
\text { aprendizaje }\end{array}$ \\
\hline & & & $\begin{array}{l}\text { Diversidad de rutas de } \\
\text { aprendizaje y secuencias } \\
\text { didácticas según el nivel real } \\
\text { del estudiantado }\end{array}$ \\
\hline & \multirow{2}{*}{ Tests } & \multirow{2}{*}{$\begin{array}{l}\text { Reglas de } \\
\text { evaluación }\end{array}$} & $\begin{array}{l}\text { Criterios de evaluación según } \\
\text { rutas de progresión }\end{array}$ \\
\hline & & & $\begin{array}{l}\text { Variedad de formatos de } \\
\text { evaluación }\end{array}$ \\
\hline
\end{tabular}

Hay, sin embargo, limitaciones a este estudio. En Nicaragua no hay un resguardo de la memoria y el acceso a la información es limitado. La falta de documentación en portales oficiales, aunado al contexto sociopolítico que se vive desde 2018 y las nuevas condiciones derivadas de la pandemia COVID-19, imposibilitó el acceso a mayores documentos que los enumerados anteriormente.

\section{Resultados y discusión}

Un lenguaje con códigos restringidos

El enfoque declarado en todos los niveles estudiados es el comunicativo funcional. La LEI se concibe como medio para comunicarse en diferentes situaciones. Esto habla de un valor instruccional que se atribuye a la lectura y la escritura. A nivel político-estratégico es evidente el énfasis que se hace en el desarrollo del lenguaje por lo que se entiende que la LEI es un medio para acceder al dominio del mismo.

La competencia comunicativa trata de desarrollar comprensiones y capacidades lingüísticas, sociolingüísticas y artísticas entendidas como capacidades globales, que permitan convertir la lengua y otras formas de expresión en un instrumento útil para la vida, significa reconocer su valor como elemento ordenador del pensamiento, que contribuye al desarrollo de las habilidades expresivas y receptivas del lenguaje y de la comunicación. (MINED, 2009, p.37). 
Sin embargo, hay una contradicción entre el enfoque declarado en el nivel político-pedagógico y las actividades de aprendizaje propuestas por niveles dependientes a este. Las actividades contenidas en el currículo no tienen una intención comunicativa, están formuladas con códigos sociolingüísticos elaborados y cuyo entendimiento requiere niveles estándares de comprensión lectora a los que no puede acceder todo el estudiantado una vez que ha ingresado a primaria. La actividad señalada a continuación, está incluida dentro de la etapa de aprestamiento, es decir, la etapa inicial del FAS con la que se supone que inicia la enseñanza convencional de la LEI. La malla orienta,

la realización del esquema gráfico con palabras, pronunciando los sonidos de las palabras, silabas y letras adecuadamente; con este ejercicio se logra el análisis y la síntesis de cada palabra, del todo a las partes y de las partes al todo. Por ejemplo:

$$
\begin{aligned}
& \text { dedo } \\
& \text { de do }
\end{aligned}
$$

Diseñar actividades que permitan la diferenciación de sonidos en palabras parecidas (pala - ola/ palmera-escalera/) (MINED, 2019a, p.73).

Esto parece problemático por cuanto no se sabe si el niño o la niña tiene dominio del código escrito porque recién llega del preescolar o porque jamás asistió a ese nivel educativo.

Al tener como eje transversal el enfoque comunicativo, el documento reconoce la diversidad y el contexto como punto de partida para alimentar el currículo, pero no parece diferenciar códigos lingüísticos elaborados y restringidos, en el sentido que lo propone Bernstein (1990); es decir, como variantes sintácticas más o menos dependientes del contexto, y que por ende se corresponden con diferentes formas de interpretar la realidad. Al no diferenciar los códigos restringidos y elaborados, se da por supuesto que el lenguaje escolar es el mismo que el del contexto de procedencia, lo que contradice lo propuesto por Bernstein (1990) ya que, tal como se expresa en el extracto anterior, la cultura de la escuela se encuentra desconectada del entorno en que las personas construyen su subjetividad.

Por ende, se está invisibilizando el contexto de procedencia. A su vez, se legitima, mediante el lenguaje, una cultura que excluye la diversidad (BOURDIEU; PASSERON, trad.2000) o bien la transforma en desigualdad escolar (PERRENOUD, 2008). Esta situación puede ser el germen de una 
contradicción entre el enfoque que declara el currículo, con las metodologías que plantea posteriormente

A nivel didáctico (MINED, 2015b, 2019c) los textos planteados están vinculados con la cultura nicaragüense, se emplean términos propios del país (por ejemplo, chagüital), incluso expresiones en miskito, que es una lengua indígena. No obstante, el texto funciona como una representación lingüística nacional hecha desde el Pacífico, para que estudiantes de esta región conozcan superficialmente costumbres y tradiciones del centro y el caribe de Nicaragua. Aunque existan menciones a otros departamentos y localidades y el texto no ofrece la posibilidad de que el niño o la niña pueda recuperar la diversidad lingüística de su contexto de procedencia.

Adicionalmente, el formato que predomina en este documento es el escrito (láminas). A manera de ejemplo véase el siguiente extracto:

[...] Un material de apoyo lo constituye el laminario que con estos fines se ha diseñado en sus primeras páginas aparecen láminas para promover el desarrollo del vocabulario, la narración y la descripción, [...]. Estas láminas pueden ser utilizadas para la descripción de lo que los niños ven, pero también para propiciar un análisis de lo que ellas sugieren, recogen escenas importantes en la vida del niño. (MINED, 2015a, pp. 9-11).

Desde el punto de vista del Diseño Universal de Aprendizaje (PILGRIM; KRIS WARD, 2017), es importante para enseñar a leer y a escribir de forma inclusiva, facilitar diversas opciones de percepción de la información, y planificar diversas formas para presentar nuevos símbolos. La diversidad social y cultural no es considerada por el currículo (DELGADO MONTOYA, 2007) ya que gran parte de la niñez nicaragüense no ha asistido al preescolar, lo que supone bajo dominio de habilidades como el concepto de impresión, conciencia fonológica o decodificación. Es decir que, aun cuando las láminas puedan evocar imágenes, su utilización consiste en redirigir al estudiantado al código escrito, cuya familiaridad o cercanía dependerá del tipo de interacción que el niño o niña haya tenido a lo largo de su vida.

Como consecuencia de lo anterior, el currículo no establece una transición ordenada de códigos restringidos a elaborados. Con base en Bernstein (1990) es posible afirmar que la construcción social del individuo está mediada por las variantes del habla que se ejercen en la familia. Por ende, al no establecer esa transición, las particularidades socioculturales del niño o niña expresadas por el habla, se vuelven un impedimento para el dominio de las habilidades de lectoescritura. Dicho de forma más sencilla, el o la estudiante que se expresa mediante códigos sociolingüísticos restringidos, parte en una situación de desigualdad por el simple hecho de hablar "diferente" a lo que la escuela espera o por no saber decodificar aún. 
Discurso instruccional con enmarcación y clasificación fuertes

Las posiciones que existen en la relación docente-estudiante son verticales, más allá de lo que declare formalmente el currículo. Es una relación basada en estímulo-respuesta. A una acción del o de la docente, una reacción del estudiantado. Ello evidencia una contradicción entre los niveles político pedagógico y metodológico, por cuanto en el Diseño Curricular (MINED, 2009), se declara la necesaria horizontalidad y los roles que deben jugar docentes y estudiantes en esa relación; pero en la malla curricular (MINED, 2019a) y las orientaciones metodológicas (MINED, 2015a), la verticalidad es lo que impera en las formas de interacción; esto se aprecia en los siguientes extractos.

Los estudiantes y docentes deben ser responsables y coparticipes del proceso enseñanza - aprendizaje, este se enriquece a través de la interacción social entre los estudiantes y entre estudiantes y docentes (MINED, 2009, p. 23).

Es importante que el maestro destaque a los alumnos por dónde empezarán el y trazado y que deben seguir la flecha y los puntos de referencia. El maestro demostrará en el pizarrón como realizar el trazado. El maestro atenderá la postura correcta de los alumnos y explicará la realización del trazado en la primera pauta. (MINED, 2015a, p. 27).

En lo referido a la flexibilidad del currículo, el MINED (2009) orienta una organización en asignaturas, mismas que, a su vez, se organizan en ámbitos de aprendizaje. El currículo también incorpora ejes transversales como medio ambiente y equidad de género, pero su estructura organizativa no evidencia formas en que esos ejes puedan fomentar la transdisciplinariedad y multidisciplinariedad que se requiere para la transversalización curricular. Con base en Bernstein y Díaz (1985), es posible afirmar que el currículo se corresponde con unos niveles de clasificación fuerte. La enseñanza de LEI, por consiguiente, es abordada desde la asignatura de Lengua y Literatura, y en el marco de esta última, es un vehículo de comunicación. Por el valor instrumental que el currículo le confiere, la LEl es un motor para promover el aprendizaje de las otras asignaturas, pero no una habilidad que pueda ser desarrollada integralmente desde todas las áreas del conocimiento.

Lo anterior es evidencia de una concepción restrictiva de flexibilidad curricular. Véase el siguiente extracto:

El Currículo será flexible en cuanto a la organización y puesta en práctica de las competencias, pues, aunque el mayor fondo de tiempo será para la implementación del currículo nacional; sin 
embargo, se priorizarán e incorporarán contenidos y competencias de relevancia y pertinencia al contexto de los centros educativos. [...]

\section{¿Cómo realizar el cálculo sobre el $70 \%$ del currículo básico nacional y el $30 \%$ a nivel local?}

- Del total de días lectivos de acuerdo al Calendario Escolar que son 200, se calcula cuanto corresponde para el desarrollo del Currículo Básico Nacional, que es el $70 \%$, con una simple regla de tres, encontraremos que son 140 días de clases.

- De igual manera el 30\% que corresponde a la adecuación del Currículo, tenemos 60 días, a los cuales restamos 11 días de los TEPCE y los Exámenes Finales que son 6 días, en total restamos 17 días, para tener un total de 43 días para contextualizar el Currículo a nivel local.

- Los 43 días del 30\% serán distribuidos por el docente en las diferentes Unidades Didácticas que vayan a desarrollar. (MINED, 2009, p. 75).

A partir de Preast, Browman y Rose, (2017) es posible afirmar que la flexibilidad para lograr la inclusión y la contextualización del currículo, se logra a partir de la adaptación del conocimiento que se quiere enseñar a las necesidades del estudiantado. Sin embargo, en el ejemplo anterior se aprecia que la unidad de contextualización son los días, lo que constituye un problema de fondo puesto que el currículo ordena que las jerarquías de abstracciones contenidas en él sean enseñadas sin considerar la integración social y material (DELGADO MONTOYA, 2007). El currículo incorpora un capital cultural (BOURDIEU; PASSERON, trad. 2000) que, a priori, es accesible a todo el estudiantado. Esa integración simbólica se realiza, no obstante, en detrimento del capital cultural propio del campo de contextualización (BERNSTEIN, 1990) del estudiantado, que no necesariamente se corresponde con la cultura impuesta en el currículo. No hay por, consiguiente, una flexibilidad con base en las particularidades culturales, lo que habla de una capital cultural innegociable contenido en el currículo. Esto, a pesar de las declaraciones formales que realizan los documentos sobre las necesidades del contexto y las experiencias previas del estudiantado. Estamos entonces frente a un arbitrio cultural (BOURDIEU; PASSERON, trad.2000). Una acción pedagógica que ejerce violencia simbólica sobre el estudiantado.

Basándonos en Frankel, Rowe, Becker y Pearson (2015) sabemos que el enfoque comunicativo se ajusta más a los parámetros de una enmarcación débil. Ello debido a las siguientes razones: 1) la LEI se concibe como una práctica cultural inserta en un contexto social concreto; 2) las habilidades que subyacen a esta competencia deben enseñarse de forma integral, interrelacionadas y ubicadas en el contexto sociocultural; 3) es necesaria la motivación intrínseca y 
4) se necesitan andamios específicos para la enseñanza de esta competencia cuya base se encuentra en la zona de desarrollo próximo y las interacciones significativas que se pueda ofrecer al estudiante para garantizar la autonomía y la progresión en el dominio.

En nuestro caso, el currículo promueve un orden inflexible y rígido para la enseñanza de la LEI. Ese orden está dado por las etapas del método FAS, que desarrolla la enseñanza de la LEI de forma lineal: primero, se desarrollan aspectos psicomotores y de discriminación auditiva por medio de la etapa de aprestamiento. Luego, se desarrolla decodificación y reconocimiento de palabras por medio de la etapa de adquisición. Finalmente, se enfoca en comprensión lectora y vocabulario por medio de la etapa de afianzamiento. Esa secuencia lineal responde a una errónea concepción de que primero se aprende a decodificar y luego a comprender; cuando, en realidad, son procesos complejos, paralelos e interrelacionados (TUNMER; HOOVER, 2019).

Anteriormente se habló de una contradicción entre el enfoque declarado y las metodologías propuestas por el currículo. Esto se aprecia en el tratamiento metodológico de la etapa de adquisición del método FAS. En primer grado, la carga horaria dedicada a esta, es de 272 horas clase, lo que corresponde a casi $63 \%$ del tiempo definido para la asignatura de Lengua y Literatura (MINED, 2019a, 2019b). A ello es necesario agregar que el mayor proceso de secuenciación didáctica se encuentra en esta etapa, mientras que las actividades de aprestamiento y afianzamiento presentan orientaciones metodológicas más imprecisas y vagas, como se podrá ver más adelante.

Adicionalmente, el desarrollo de la etapa de adquisición se realiza de una forma desvinculada de las prácticas de cultura escrita de niños y niñas. El proceso es mecánico y repetitivo y se basa en la asociación fonema-grafema, su reconocimiento en las palabras, y su trazado en el cuaderno de escritura.

Una vez seleccionada la palabra el maestro debe pronunciarla, teniendo en cuenta su correcta articulación, para que los niños distingan el sonido con precisión y puedan reproducirlo correctamente con posterioridad. Esta demostración del análisis de la palabra junto con el trazado del esquema deberá repetirse tantas veces como se estime necesario. Después los niños dirigidos por el maestro, harán el análisis fónico y el trazado del esquema gráfico, hasta lograr una independencia cada vez mayor. Esta parte de la metodología tiene como objetivo demostrar si los alumnos se apropiaron correctamente de los conocimientos que han recibido, si identifican las grafías estudiadas y si establecen la correspondencia adecuada. [...] El maestro presentará a los alumnos una palabra que contenga el sonido estudiado y colocará en un lugar visible las letras de imprenta minúscula y mayúscula que lo representa. Mostrará la grafía cursiva minúscula y les dirá que esa es la forma de la letra con que van a escribir, colocará o trazará esta letra cursiva al 
lado de la de imprenta, y pedirá a los alumnos que digan en qué se parecen y en qué se diferencian. Una vez establecida esta comparación, presentará la mayúscula cursiva y la colocará debajo de la minúscula y al lado de la de imprenta, compararán las dos letras cursivas entre sí, destacarán sus semejanzas y diferencias y compararán las mayúsculas cursivas y de imprenta. El maestro será muy cuidadoso en el trazado de las letras en tarjetas, carteles y en el pizarrón. (MINED, 2015a, pp. 24-26).

Con base en lo anterior es posible afirmar que el currículo propone actividades altamente reguladas, jerarquizadas y aisladas del campo de contextualización del estudiantado. Además, estas no promueven el desarrollo de competencias y pensamiento complejo debido al débil tratamiento metodológico de las habilidades amplias (PARIS, 2005). En consecuencia, el currículo desconoce lo que dicta la evidencia respecto a la enseñanza explícita (ALLINGTON, 2002) para estas habilidades, y al enfoque balanceado entre decodificación y comprensión (GUARDIA, 2018).

A nivel didáctico, los documentos revisados (MINED,2019c, 2015b) parecen reforzar los lineamientos de la malla curricular (MINED, 2019a, 2019b) y las orientaciones metodológicas (MINED, 2015a). Los documentos se limitan exclusivamente a cumplir con las etapas del método FAS. El libro de aprestamiento no tiene un enfoque para la enseñanza expresado en el currículo. Se promueve el uso del lenguaje oral, así como el análisis fónico y el fortalecimiento del trazo.

El contenido del libro de texto, que trabaja en función de las etapas de adquisición y aprestamiento, se limita a presentar vocales y consonantes; que están ordenadas según grupos fonemáticos. Más adelante, en la etapa de afianzamiento, se presentan pequeños textos con el fin de reforzar la lectura. Lo llamativo es que los ejercicios de este libro no ofrecen mayores indicaciones. Los textos a leer, por ejemplo, están desprovistos de un contexto específico, o de orientaciones metodológicas claras. Véase los ejemplos extraídos del libro de texto (MINED, 2019c).

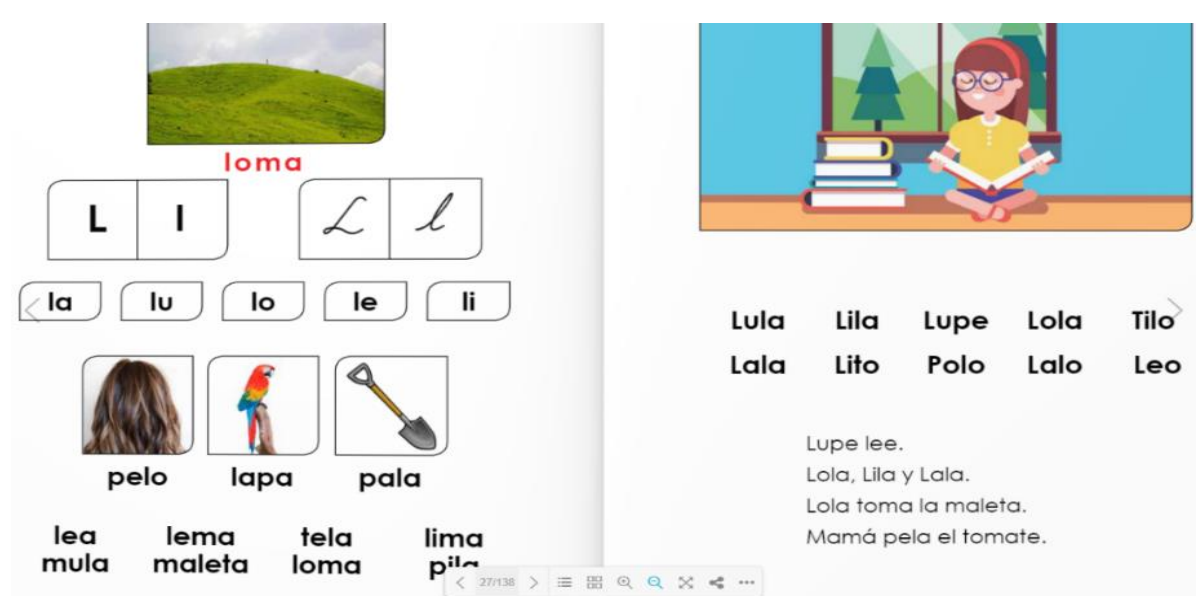

Fuente: MINED 


\section{Un discurso instruccional limitado y excluyente}

Las actividades de aprendizaje no están estructuradas, son muy amplias y vagas, carecen de tratamiento metodológico. Asimismo, carecen de variedad, y claramente no están orientadas por un objetivo de comunicación o vinculadas a aspectos de la vida de los estudiantes que tengan sentido y significado para ellos como establece el enfoque comunicativo (FRANKEL, ROWE, BECKER; PEARSON,2015). La única variación que se identifica son los tipos de textos a emplear, pero la dinámica se mantiene. A continuación, un ejemplo:

\section{LECTURA EN EL LIBRO DE TEXTO}

Esta parte de la metodología tiene como objetivo demostrar si los alumnos se apropiaron correctamente de los conocimientos que han recibido, si identifican las grafías estudiadas y si establecen la correspondencia adecuada. Este momento permite la realización de diferentes actividades:

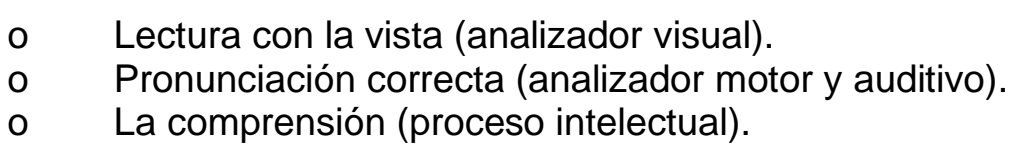

Estas actividades deben propiciar que de manera amena y divertida los niños formen y lean palabras, que todos los alumnos lean para poder comprobar su avance, que se de tratamiento a la comprensión de todas las palabras del texto. Para esto podemos emplear los siguientes procedimientos:

- Identificar el significado de una palabra por medio de un objeto, una ilustración

- Seleccionar un sonido dado.

- Utilizar la palabra en una oración.

Todas las actividades que se realicen en cada uno de los momentos, deben responder a las características del fonema y del grafema objeto de estudio, a las características del grupo, a su avance y a la experiencia del maestro. (MINED, 2015a, p.24).

Los documentos revisados no ofrecen una verdadera progresión en el dominio de las habilidades de la LEI entre diferentes grados y menos entre diferentes momentos del mismo año académico (ALLINGTON, 2002), con lo que se obstaculiza el progreso del aprendizaje que requiere desafíos cada vez más complejos. Esto se evidencia en la nula diferenciación que existe entre competencias definidas para primero y segundo grado:

Competencias de primer grado: 1. Comprende los textos expositivos y literarios leídos. 2. Evidencia vinculación personal con los textos literarios. 3. Aplica la escritura correcta de palabras en la redacción de enunciados. Competencias de 
segundo grado: Comprende los textos expositivos y literarios leídos de manera oral y silenciosa. 2. Evidencia vinculación personal con los textos literarios. 3. Emplea la forma verbal conjugada en la comunicación de mensajes escritos por medio de la oración simple. 4. Expresa mensajes orales atendiendo a las normas de conversación. (MINED, 2019b, p. 49).

Tampoco se evidencian procesos metodológicos que respeten los diferentes niveles de aprendizaje de niños y niñas mediante el uso de diversos formatos de procesamiento de información y expresión de aprendizajes (KRIS WARD; PILGRIM, 2017). Los documentos no ofrecen mayores pautas para intervenir en casos de estudiantes con retrasos en cuanto al nivel de aprendizaje.

Fundamentalmente se seguirá brindando atención a los alumnos que no han desarrollado en forma suficiente, las habilidades para establecer la correspondencia fonema-grafema. Además, se continuará trabajando en primer término para lograr la síntesis al leer palabras y para trabajar las cualidades de la lectura: corrección, comprensión, fluidez y expresividad, dando por supuesto mayor énfasis a las dos primeras con un sistema de actividades encaminadas al logro de esos fines. Se combinarán procedimientos utilizados en la etapa de adquisición (formación de palabras y oraciones, trabajo en el componedor, análisis de palabras si fuera necesario), con otras formas de trabajo que permitan lograr el desarrollo de las habilidades que se requieran en esta etapa: debe predominar la lectura del alumno de diversas formas y se brindará la debida atención a la expresión oral. (MINED, 2015a, p. 40).

Con base en este extracto, se puede ver como el currículo ordena poner atención al nivel que se está desarrollando en niños y niñas, pero básicamente el o la docente está obligado/a a hacer más de lo mismo cuando se presentan dificultades de aprendizaje en el estudiantado, siempre con el mismo formato escrito.

En lo que se refiere a los criterios de selección de materiales, solo se contemplan aquellos definidos por el método FAS: Libro de aprestamiento, libro de texto, cuaderno de escritura, componedor individual y colectivo, tarjetas y láminas de vocabulario o letras, nada que provenga del medio o de las familias o que pueda ser innovado por las/os maestras/os. En el documento de orientaciones metodológicas se concibe al manual de texto como un medio insustituible.

El libro resulta un medio insustituible para el desarrollo de las clases, porque le ofrece al maestro un material organizado en orden ascendente de dificultades, teniendo en cuenta las características del niño de este grado y sus intereses. (MINED, 2015a, p. 16). 
Con base en Allington (2002) y Kris Ward y Pilgrim (2017), es posible afirmar que las actividades de aprendizaje no conciben la inclusión dentro de los procesos educativos, lo que se traduce en actividades de mero trámite, en formatos únicos, con ritmos y secuencias uniformes y de talla única.

En lo referido a las actividades de evaluación, las mismas se confunden con actividades de aprendizaje, por lo que no ofrecen indicadores para medir el aprendizaje, sino la memorización sin razonamiento de la lección. Sirva de ejemplo la siguiente cita:

Lea diversos textos, identifica palabras con sílabas mixtas terminadas en $\mathrm{p}$ y vocales dobles.

- Elabora una lista de palabras identificadas de la lectura.

- Escriba palabras con sílabas mixtas terminadas en $\mathrm{p}$ y vocales dobles y redacta oraciones.

- Lea diversos refranes y adivinanzas.

- Establece un conversatorio con los compañeros y determina el mensaje, luego lo presenta en plenario". (MINED, 2019b, p.56).

Cuando se orientan este tipo de prácticas, el currículo falla al momento de crear evaluaciones formativas, orientadoras y enfocadas en los aprendizajes por cuanto no son posibles de crear espacios protegidos (BOURGEOIS, 2009) en los cuales los y las niñas no tengan miedo de cometer errores por cuanto esta acción supone un paso necesario para el aprendizaje.

El discurso instruccional es, paradójicamente, contradictorio con lo que plantea el currículo sobre el enfoque adoptado (comunicativo), pero es consecuente con el discurso regulativo que subyace más allá de lo explícito o literal. Los niveles fuertes de enmarcación y clasificación se corresponden con las reglas de selección, ritmo y evaluación definidas en el currículo (BERNSTEIN, 1990). Esto, a pesar de las constantes declaraciones en el currículo respecto a las relaciones horizontales, o la recuperación de experiencias previas. El currículo no ofrece alternativas ni promueve la libre elección y la autonomía de aprendizaje (PILGRIM; KRIS WARD, 2017). Todo lo contrario, ofrece una ruta única para el dominio de la LEI. Todas las actividades, contenidos y palabras son seleccionados y definidos por el magisterio. Los criterios para seleccionar las actividades son prácticamente inexistentes y se solicita al alumnado que vaya a un ritmo específico y uniformador.

\section{CONCLUSIONES}


En definitiva, el discurso pedagógico que subyace del Método FAS se corresponde con los lineamientos de la reproducción cultural (BOURDIEU; PASSERON, trad. 2000). El currículo nacional integra simbólicamente un capital cultural (DELGADO MONTOYA, 2007) accesible a todos y todas. Este no obstante se hace presente en detrimento de la cultura propio del campo de contextualización del sujeto. Es decir, para acceder a él, el o la estudiante deberá desconocer y olvidar su propio contexto, lo que supone para él o ella, una transición desconcertante de códigos sociolingüísticos restringidos a elaborados.

Por consiguiente, el método FAS, como componente esencial del diseño curricular nicaragüense, legitima un arbitrio cultural que excluye a niños y niñas del aprendizaje significativo de la LEI. Al realizar el contraste del discurso pedagógico que subyace a él, nos encontramos con que se ajusta con los lineamientos de aquel discurso que ve en la LEI una herramienta puesta al servicio del mercado y la modernización (MOUNT-CORS, 2016).

Desde esta lógica, es un discurso pedagógico en donde existe un lenguaje "target" al cual acceder para entrar en las dinámicas globales, lo que no necesariamente supone algo negativo en la medida que se garantice inclusión en el acceso a ese lenguaje. El problema de fondo es que el aprendizaje de la LEI responde a intereses de otros agentes reguladores que no parecen corresponderse con las necesidades de aprendizaje de niños y niñas.

Este discurso pedagógico legitima igualmente la enseñanza homogénea, y centralizada en la figura del profesorado. Es también una enseñanza que se basa exclusivamente en el uso de manuales, sin considerar otros recursos que el entorno brinda. Los niveles de enmarcación y clasificación en el currículo son fuertes, sin embargo, a nivel declarativo, este afirma estar alineado con el enfoque comunicativo. Es importante enfatizar que la nula intencionalidad comunicativa de las actividades de aprendizaje, así como la inexistente posibilidad de flexibilizar las estrategias y hacerlas significativas para el estudiantado, son claros indicadores que el enfoque comunicativo no es más que una declaración vacía y carente de realización metodológica.

En definitiva, se aprecia un currículo que es agente de la exclusión educativa. Un currículo que, si no empieza a revisarse su contenido con base los parámetros de un discurso pedagógico transformador, seguirá ensanchando las brechas históricas de equidad en educación. Finalmente, se considera importante profundizar en los hallazgos de este estudio mediante una investigación más de tipo etnográfica para comprender cómo el discurso pedagógico subyacente a la implementación del método FAS se expresa en las aulas de clase. Estas líneas de discusión podrán brindar insumos para calcular mejor los pasos hacia la educación con calidad y equidad.

\section{REFERENCIAS BIBLIOGRÁFICAS}


APPEL, M. W. What is present and absent in critical analyses of Neoliberalism in Education. Peabody Journal of Education, 1 (6), 1-6, 2017. Recuperado de https://DOI:10.1080/0161956X.2016:1265344.

APPLE, M.W. Ideology and Curriculum. (4.Ed.) New York: Routledge Taylor \& Francis Group, 2019.

ASTETE BARRENECHEA, C. Bourdieu: el poder en el campo de la educación y la pedagogía. Horizonte de la ciencia, 7 (12), 223-239, 2017. Recuperado de https://www.researchgate.net/publication/318874492_Pierre_Bourdieu_el_poder_en_el _campo_de_la_educacion_y_la_pedagogia.

BANERJEE, A. \& DUFFLO, E. Repensar la pobreza. Un giro radical en la lucha contra la desigualdad global. México D.F.: Santillana Ediciones Generales S.A. de C.V., 2012.

\section{BERNSTEIN, B. \& DÍAZ, M. Hacia una teoría del discurso pedagógico,1985}

Recuperado de

https://www.academia.edu/2098425/Hacia_una_teor\%C3\%ADa_del_discurso_pedag\% C3\%B3gico.

BERNSTEIN, B. Clases sociales, lenguaje y socialización, 1975a. Recuperado de revistas.pedagogica.edu.co/index.php/RCE/article/download/5117/4196.

BERNSTEIN, B. Clasificación y enmarcación del conocimiento educativo, 1975b. Recuperado de http://www.pedagogica.edu.co/storage/rce/articulos/15_06ens.pdf.

BERNSTEIN, B. Clases sociales y pedagogías: visibles e invisibles. 1975c.

Recuperado de https://www.infoamerica.org/documentos_pdf/bernstein06.pdf.

BERNSTEIN, B. Códigos elaborados y restringidos: Visión General y Crítica. En BERNSTEIN, B (autor). La estructura del Discurso Pedagógico,.100-126, Madrid: Editorial Morata, 1990.

BOURDIEU, P. \& PASSERON, J.C. Reproduction in Education, Society and Culture. (2.ed.). London: Sage Publications, 2000.

BOURGEOIS, E. Apprentissage, motivation et engagement en formation. Education permanente, (136), 101-109, 2009.

CASTILLO, M. ELVIR, A.P. \& VIJIL, J. Prioridades de la Educación nicaragüense para el siglo XXI. Managua: CIASES, 2016.

DELGADO MONTOYA, W. Inclusión: Principio de calidad educativa desde la perspectiva del desarrollo humano. Revista Educación, 31 (2), 41-56, 2007. Recuperado de https://www.researchgate.net/publication/26548936_Inclusion_principio_de_calidad_ed ucativa_desde_la_perspectiva_del_desarrollo_humano.

FRANKEL, K.K., BECKER, B.L.C., ROWE, M.W. \& PEARSON, P.D. From "What is reading?" to What is literacy? Journal of Education, 196 (3), pp. 7-17, 2015

FREIRE, P. Pedagogía del oprimido. (2.Ed.) Madrid: Siglo XXI de España Editores S.A., 2012. 
GUTIÉRREZ-BRAOJOS, C. \& SALMERÓN PÉREZ, H. Estrategias de comprensión lectora: enseñanza y evaluación en educación primaria. Profesorado. Revista de Currículum y Formación de Profesorado, 16 (1), 183-202, 2012. Recuperado de https://www.redalyc.org/pdf/567/56724377011.pdf.

HOOKS, B. Teaching to transgress. Education as the practice of Freedom. New York: Routledge Taylor \& Francis Group, 1994.

HOOKS, C. Teaching community. A pedagogy of hope. New York: Routledge Taylor \& Francis Group, 2003.

HOOKS, B. Teaching critical thinking. Practical Wisdom. New York: Routledge Taylor \& Francis Group, 2010.

INSTITUTO DE ESTUDIOS ESTRATÉGICOS Y POLÍTICAS PÚBLICAS (IEEPP). Eficacia escolar y calidad educativa en Nicaragua. ¿Qué nos dicen los resultados del TERCE? Managua: Autor, 2016.

JACOBS, M.M. Literacy, Education and Inequality: assimilation and resistance narratives from families residing at a homeless shelter. Critical questions in Education (Special Issue), 5 (3), pp. 172-188, 2017. Recuperado de https://files.eric.ed.gov/fulltext/EJ1046708.pdf.

KIM, Y.-S. G., BOYLE, H. N., ZUILKOWSKI, S. S., \& NAKAMURA, P. Landscape Report on Early Grade Literacy. Washington, D.C.: USAID, 2016.

MINISTERIO DE EDUCACIÓN (MINED) Método Fónico Analítico Sintético. Orientaciones metodológicas. Managua: Autor, 2015a.

MINISTERIO DE EDUCACIÓN. Cuaderno de aprestamiento. Managua: Autor, 2015b.

MINISTERIO DE EDUCACIÓN. Mallas curriculares de primer ciclo de educación primaria. Autor: Managua, 2019a.

MINISTERIO DE EDUCACIÓN. Primera unidad pedagógica. Primaria Regular. Grado: Primero y Segundo Grado. Segundo Semestre. Managua: Autor, 2019b.

MINISTERIO DE EDUCACIÓN. Lengua y literatura. Primer grado. Libro de lectura. Autor: Managua, 2019c.

MINISTERIO DE EDUCACIÓN. Currículo Nacional Básico: Diseño Curricular del Subsistema de la Educación Básica y Media Nicaraguense. Managua: Autor, 2009.

MOUNT CORS, M.F. Theoretical frameworks: building a cultural model for literacy. En MOUNT-CORS, M.F. (autora). Global Literacy in Local Learning Contexts. (17-51). New York: : Routledge Taylor \& Francis Group, 2016.

PARIS, S. G. Reinterpreting the development of reading skills. Reading Research Quarterly, 40 (2). 184-202, 2005.

PERRENOUD, J. La construcción del éxito y del fracaso escolar. (4.Ed.) Madrid: Ediciones Morata, S.L., 2008. 
PETERSEN, A.J. Intersectionality and the construction of inclusive schools. En CURRAN, C.M.(Ed.), \& PETERSEN, A.J. (Ed.) Handbook of Research on Classroom Diversity and Inclusive Education Practice. (1-26). USA: IGI Global, 2017.

PILGRIM, J. \& KRIS WARD, A. Universal Design for Learning: A framework for supporting Effective Literacy Instruction. En CURRAN, C.M. (Ed.), \& PETERSEN, A.J. (Ed.) Handbook of Research on Classroom Diversity and Inclusive Education Practice. (282-310). USA: IGI Globa, 2017.

PREAST, J.L., BOWMAN, N. \& ROSE, C.A. Creating inclusive classrooms communities through social and emotional learning to reduce social marginalization among students. En CURRAN, C.M.(Ed.), \& PETERSEN, A.J. (Ed.) Handbook of Research on Classroom Diversity and Inclusive Education Practice. (183-200). USA: IGI Global, 2017.

PROGRAMA DE CAPACIDADES LAC READS. Early Grade Reading in Latin America: A Sistematic Review, 2016.

SALAZAR PÉREZ, M. \& SAAVEDRA, C.M. A call for onto-epistemological diversity in early childhood education and care: centering global south conceptualizations of childhood, Review of education, (41), 1-29, 2017. Recuperado de https://DOI:10.3102/0091732X16688621.

WAGNER, D., MURPHY, K. \& DE KORNE, H. Learning First: a research agenda for improving learning in low-income countries. USA: Center for Universal Education, 2012. 